\title{
Características de qualidade para substâncias inorgânicas em água mineral envasada comercializada na cidade de São Paulo/SP
}

Quality characteristics for inorganic substances in bottled mineral water sold in the city of São Paulo/SP

\author{
L. J. de Arauz*; M. L. Buzzo; L. R. V. Mataveli; M. F. H. Carvalho \\ Núcleo de Contaminantes Inorgânicos, Centro de Contaminantes, Instituto Adolfo Lutz, 01246-000, São Paulo-SP, \\ Brasil \\ *luciana.arauz@ial.sp.gov.br \\ (Trabalho avaliado e selecionado pela Comissão do III CTOCTA)
}

\begin{abstract}
A água mineral é um recurso proveniente de fonte natural e importante à saúde humana, por possuir características químicas e físico-químicas em sua composição necessárias para a manutenção do equilíbrio adequado de diversos processos bioquímicos e mecanismos fisiológicos que ocorrem no organismo humano. Ainda, existe uma preocupação com a ingestão de contaminantes inorgânicos, de origem natural ou antropogênica, uma vez que esses elementos, mesmo em baixas concentrações, podem ser tóxicos e representar um problema de Saúde Pública. Portanto, é primordial avaliar a qualidade das águas minerais que são disponibilizadas para consumo da população, em atendimento à legislação Resolução-RDC 274/2005, quanto aos níveis de metais e semimetais. Este trabalho teve como objetivo realizar a quantificação dos elementos químicos Al, $\mathrm{Ag}, \mathrm{As}, \mathrm{Ba}, \mathrm{Be}, \mathrm{Ca}, \mathrm{Cd}, \mathrm{Cr}, \mathrm{Cu}, \mathrm{K}, \mathrm{Mg}, \mathrm{Mn}, \mathrm{Na}, \mathrm{Ni}, \mathrm{Se}, \mathrm{Pb}, \mathrm{Tl}$ e Zn, em 10 amostras de águas minerais envasadas de procedência nacional, coletadas pela Vigilância Sanitária do Estado de São Paulo no comércio local, como parte integrante do Programa Paulista de Alimentos, entre 2018 e 2019, por Espectrometria de Massas por Plasma Indutivamente Acoplado. Para os elementos previstos na legislação vigente, foram obtidos resultados satisfatórios para todas as amostras de águas minerais analisadas. Para os demais analitos avaliados, os níveis obtidos também estavam abaixo dos valores preconizados pelas regulamentações internacionais. Os resultados obtidos neste estudo indicaram que as águas minerais das diferentes marcas comercializadas na cidade de São Paulo são adequadas e seguras para o consumo da população, em relação aos parâmetros avaliados.
\end{abstract}

Palavras-chave: água mineral, metais, ICP-MS.

Mineral water is a natural resource important to human health, as it has chemical and physicochemical characteristics in its composition that are necessary to maintain the proper balance of various biochemical processes and physiological mechanisms that occur in the human body. Still, there is a concern with the ingestion of inorganic contaminants, of natural or anthropogenic sources, since these elements, even at low concentrations, can be toxic and a Public Health concern. Therefore, it is essential to assess the quality of mineral waters available for consumption by the population, in compliance with the RDC 274/2005 legislation, regarding the levels of metals and semi-metals. This work aimed to carry out the quantification of the chemical elements $\mathrm{Al}, \mathrm{Ag}, \mathrm{As}, \mathrm{Ba}, \mathrm{Be}, \mathrm{Ca}, \mathrm{Cd}, \mathrm{Cr}, \mathrm{Cu}, \mathrm{K}, \mathrm{Mg}, \mathrm{Mn}, \mathrm{Na}, \mathrm{Ni}, \mathrm{Se}, \mathrm{Pb}, \mathrm{Tl}$ and $\mathrm{Zn}$, in 10 samples of bottled mineral waters of national origin, collected by the São Paulo Sanitary Surveillance in local markets, as part of the São Paulo Food Program, between 2018 and 2019, using Inductively Coupled Plasma Mass Spectrometry. For the elements comprised in the current legislation, satisfactory results were obtained for all mineral water samples analyzed. For the other analytes evaluated, the levels were also below the values recommended by international regulations. The results obtained in this study indicated that bottled mineral waters from different brands sold in the city of São Paulo are suitable and safe for the population to consume, regarding the parameters evaluated.

Keywords: mineral water, metals, ICP-MS.

\section{INTRODUÇÃO}

A ingestão de água mineral tem grande relevância para a saúde devido à presença de sais minerais em sua composição, que são essenciais para os seres humanos devido à sua importância para o funcionamento fisiológico do organismo. Os minerais são compostos inorgânicos que estão 
presentes na água sob forma iônica e por isso são facilmente absorvidos pelo trato gastrointestinal. Sua ingestão adequada promove a distribuição dos nutrientes pelos diferentes órgãos do corpo, auxilia na regulação da temperatura corpórea, elimina as toxinas por meio da urina e da transpiração e estimula o trânsito intestinal $[1,2]$.

A água mineral é proveniente de formação subterrânea e pode fluir naturalmente para a superfície da terra sob a forma de fontes naturais ou, mais comumente, são captadas artificialmente por meio de perfuração de poços tubulares. É formada quando as águas das chuvas penetram no solo e atravessam diversas camadas até atingirem camadas impermeáveis, onde se depositam. Nesse trajeto na parte inferior do solo, a água passa por várias rochas contendo substâncias minerais, como carbonato e sulfato de cálcio, que se diluem na água promovendo o seu enriquecimento. A água ao atravessar as superfícies de rochas tem suas moléculas orgânicas filtradas, e por isso apresenta baixo conteúdo de compostos orgânicos [3]. Sua composição varia de acordo com as rochas e terrenos pelos quais ocorre a infiltração no solo, podendo também ter contribuição do clima, biota, entre outras [4]. Após o envase da água destinado ao consumo humano, o produto final para comercialização é considerado, em termos legais, como alimento e a inspeção do processo de produção visando a qualidade do produto final é regulamentada pela Agência Nacional de Vigilância Sanitária (ANVISA) [5].

Por outro lado, deve-se considerar ainda a vulnerabilidade à contaminação a que as fontes de água natural ou subterrânea estão sujeitas, provocadas possivelmente por ação de origem antrópica [6-8]. Assim, os contaminantes inorgânicos que podem estar presentes na água são caracterizados como potencialmente tóxicos e prejudiciais à saúde do organismo por possuírem elevada toxicidade e não apresentarem função biológica conhecida no organismo, como: Al, As, $\mathrm{Cd}$ e $\mathrm{Pb}$, entre outros. Estes elementos classificados como não essenciais, podem ocasionar diversos agravos à saúde quando são ingeridos, como: efeitos neurológico, vascular, respiratório, sistema reprodutor e trato gastrintestinal; bem como lesão dérmica, câncer, entre outros distúrbios [9-11].

Neste sentido, considerando a possível alteração das propriedades microbiológicas e físicoquímicas da composição das águas minerais envasadas destinadas ao consumo humano, torna-se primordial monitorar sua qualidade, na verificação do atendimento aos padrões de identidade e qualidade discriminados em legislação [5] com relação aos limites máximos permitidos para as substâncias, e que podem representar risco à saúde. O estabelecimento de medidas de correção pode ser aplicado quando o monitoramento indicar que os limites críticos obtidos nas avaliações foram excedidos aos preconizados em legislação, permitindo inferir na tomada de ações pelos órgãos competentes que fiscalizam esse tipo de produto ofertado e consumido pela população em decorrência da promoção da Saúde Pública.

Dentro deste contexto, o Núcleo de Contaminantes Inorgânicos (NCI) do Instituto Adolfo Lutz (IAL) atua nas ações laboratoriais em conjunto com o sistema de Vigilância Sanitária, participando do Programa Paulista de Alimentos [12] na avaliação das concentrações de elementos inorgânicos (metais e semimetais) em alimentos que possam causar efeitos prejudiciais à saúde da população do Estado de São Paulo, por emprego de técnicas espectrométricas. Este Programa tem como meta monitorar a qualidade dos produtos alimentícios disponíveis no comércio e também a qualidade sanitária dos estabelecimentos que os comercializam, aplicando a legislação sanitária, identificando setores do comércio/indústria de alimentos que necessitam de intervenção preventiva, quando pertinente. Dentre os tipos de produtos avaliados e disponibilizados à aquisição no comércio, destaca-se a água mineral envasada.

$\mathrm{O}$ atendimento aos reduzidos valores permitidos pela legislação brasileira para nutrientes e contaminantes inorgânicos nesse tipo de produto requer a utilização de técnica analítica instrumental como a Espectrometria de Massas por Plasma Indutivamente Acoplado (ICP-MS), configurada com uma das mais adequadas para a quantificação de metais e semimetais em águas destinadas ao consumo humano. Essa instrumentação analítica multielementar é amplamente empregada na determinação simultânea de cerca de 70 elementos-traços devido à alta sensibilidade, detecção em baixos níveis de concentração e rapidez na obtenção de resultados, quando comparada a técnicas monoelementares, como a Espectrometria de Absorção Atômica [13]. 
Desta forma, face ao crescente consumo da água mineral envasada e em cumprimento parcial ao Programa Paulista de Alimentos, este estudo teve como objetivo avaliar o padrão de qualidade química na determinação dos metais e semimetais ( $\mathrm{Al}, \mathrm{Ag}, \mathrm{As}, \mathrm{Ba}, \mathrm{Be}, \mathrm{Ca}, \mathrm{Cd}, \mathrm{Cr}, \mathrm{Cu}, \mathrm{K}, \mathrm{Mg}$, $\mathrm{Mn}, \mathrm{Na}, \mathrm{Ni}, \mathrm{Se}, \mathrm{Pb}, \mathrm{Tl}$ e $\mathrm{Zn}$ ), por Espectrometria de Massas por Plasma Indutivamente Acoplado (ICP-MS), em amostras de águas minerais envasadas tradicionalmente distribuídas no mercado nacional, sem gás e sem adição de sais, comercializadas na cidade de São Paulo em atendimento à Resolução-RDC 274/2005 [5] que fixa a identidade para águas envasadas e gelo, bem como fornece ferramenta às autoridades sanitárias para tomada de ação preventiva, quando necessário.

\section{MATERIAL E MÉTODOS}

\subsection{Amostras}

Um total de 10 garrafas de água mineral natural acondicionadas em embalagens plásticas, do tipo sem gás e sem adição de sais, de procedência de fontes naturais nacionais, constituídas de diferentes marcas tradicionalmente distribuídas no mercado nacional e nomeadas de A a J, foram coletadas pelos Grupos de Vigilância Sanitária do Estado de São Paulo [14, 15] no comércio da cidade de São Paulo, entre 2018 e 2019, como parte integrante do Programa Paulista de Alimentos. As amostras foram abertas no momento da análise e acidificadas com ácido nítrico ultrapuro a $0,2 \%(\mathrm{v} / \mathrm{v})$ e homogeneizadas, não sendo submetidas a qualquer pré-tratamento antes da análise. Amostras de águas cujas concentrações dos elementos determinados estavam compreendidas na faixa linear da curva analítica foram analisadas diretamente. Já para aquelas que apresentaram concentrações superiores à faixa de trabalho, foram diluídas apropriadamente para ajuste ao intervalo da curva analítica. As determinações dos elementos foram realizadas em duplicata e dentro do prazo de validade do produto.

\subsection{Reagentes e materiais}

Todas as soluções foram preparadas com água ultrapura com resistividade de $18,2 \mathrm{M} \Omega . \mathrm{cm}$ (Sistema Milli-Q ${ }^{\circledR}$, Merck Millipore, modelo Integral 10) e ácido nítrico de grau ultrapuro (Suprapur ${ }^{\circledR} 65 \%$, Merck).

Os materiais plásticos de polipropileno utilizados para o preparo das soluções de uso laboratorial foram previamente descontaminados em banho de solução de detergente neutro a 2 $\%$ (v/v) (Extran, Merck), seguido da imersão em banho contendo solução de ácido nítrico (P.A., $65 \%$, Merck) a $20 \%$ (v/v) por 24 horas, enxaguados em água ultrapura e secos à temperatura ambiente, sob fluxo laminar localizado em área limpa com controle de material particulado.

Soluções padrão monoelementares com concentrações de 1.000 ou $10.000 \mathrm{mg} / \mathrm{L}$ (Inorganic Ventures ${ }^{\circledR}$ e NSI Labs Solutions) foram diluídas para o preparo da curva analítica, em solução de ácido nítrico grau ultrapuro com concentração final de $0,2 \%(\mathrm{v} / \mathrm{v})$, incluindo o branco dos reagentes.

Solução de padrão interno contendo $5 \mu \mathrm{g} / \mathrm{L}$ dos elementos $\mathrm{Ge}$, In, Re e Sc, foi adicionada em linha a todas as soluções e amostras analisadas, para normalizar as contagens do detector do instrumento em uma escala absoluta e para corrigir qualquer desvio de sinal.

\subsection{Controle interno e externo da qualidade}

Materiais de Referência Certificados NIST SRM 1643f Trace elements in water e NIST SRM 1640a Trace elements in natural water foram utilizados no controle interno da qualidade em cada lote de análise. Os resultados foram reportados em porcentagens de recuperação e avaliados de acordo com a faixa de aceitação de 80 a $100 \%$, de acordo com o documento da AOAC [16], para a faixa de concentração em estudo. Adicionalmente, também foram elaboradas cartas controles para o branco dos reagentes, com intervalo de aceitação adotado pelo laboratório de $\pm 20 \%$. 
Para assegurar a confiabilidade metrológica das medições analíticas, o controle externo da qualidade foi efetuado com participações periódicas do laboratório em Programa Interlaboratorial e Ensaio de Proficiência, em âmbito nacional, realizados pela Companhia de Saneamento Básico do Estado de São Paulo (SABESP) e Serviço Nacional de Aprendizagem Industrial (SENAI) para a determinação de metais e semimetais em águas potáveis, respectivamente [17].

\subsection{Metodologia Analítica}

A metodologia analítica para a determinação dos metais e semimetais em amostras de águas minerais em atendimento à legislação vigente [5] foi adaptada seguindo as recomendações do Método EPA 200.8 [18]. Os ensaios para a quantificação dos elementos inorgânicos em amostras de água realizadas pelo NCI/IAL são acreditados pela Coordenação Geral de Acreditação do Instituto Nacional de Metrologia, Qualidade e Tecnologia (CGCRE/INMETRO) em cumprimento aos requisitos gerenciais e técnicos da Norma ABNT NBR ISO/IEC 17.025 [19], com selo de acreditação CRL 0679 [20].

Para a avaliação dos elementos Al, Ag, As, Ba, Be, Ca, Cd, Cr, Cu, K, Mg, Mn, Na, Ni, Se, $\mathrm{Pb}$, Tl e $\mathrm{Zn}$ foi utilizado o Espectrômetro de Massas por Plasma Indutivamente Acoplado (ICPMS, modelo ELAN DRC-II, marca Perkin Elmer).

Para evitar contaminação química, os manuseios das amostras de água mineral e das soluções padrão utilizadas na curva analítica foram executados em ambiente com controle de material particulado (sala limpa ISO Classe 7) em área limpa ISO Classe 7 e com temperatura controlada de $20 \pm 2{ }^{\circ} \mathrm{C}$.

\section{RESULTADOS E DISCUSSÃO}

Devido ao crescimento industrial, comercialização e consumo de água mineral envasada em diversas regiões do Brasil [7, 21-23], torna-se de grande importância o controle do seu padrão de identidade e qualidade, a fim de garantir a segurança da saúde da população. Pesquisas na literatura mostram que, em uma época caracterizada por uma forte poluição química, potencialmente por ação antrópica [24-28], os consumidores ainda consideram a água mineral envasada como símbolo de saúde e a prática de seu uso no cotidiano [29-31].

Diante da relevância deste estudo, a Tabela 1 apresenta os níveis de concentração de nutrientes e de contaminantes inorgânicos (metais e semimetais) encontrados em amostras de águas minerais envasadas tradicionalmente distribuídas no mercado nacional, sem gás e sem adição de sais, de procedência nacional, de diferentes marcas e lotes, coletadas em diferentes locais de comércio pelo Grupo de Vigilância Sanitária, para a verificação de sua conformidade com a legislação vigente.

De acordo com os resultados obtidos para os elementos químicos $\mathrm{As}, \mathrm{Ba}, \mathrm{Cd}, \mathrm{Cr}, \mathrm{Cu}, \mathrm{Mn}, \mathrm{Ni}$, $\mathrm{Se}$ e $\mathrm{Pb}$, observou-se que em todas as amostras de águas minerais avaliadas as concentrações estavam abaixo dos valores máximos permitidos (VMP) em legislação [5], ou ainda os níveis encontraram-se abaixo do limite de quantificação do método analítico. Portanto, os produtos avaliados para os ensaios dos nove elementos citados acima, estão em conformidade com a legislação em vigor, indicando a provável ausência de contaminação originada principalmente por ação antrópica e, consequentemente, consideradas satisfatórias para o consumo da população na cidade de São Paulo.

Ainda, outros parâmetros avaliados de contaminantes inorgânicos não previstos em legislação, e que podem representar riscos à saúde da população quando presentes em níveis prejudiciais à saúde humana, também foram determinados: $\mathrm{Ag}, \mathrm{Al}, \mathrm{Be}$, Tl e $\mathrm{Zn}$. De forma semelhante aos resultados anteriores, em todos os casos os valores de concentração desses metais encontraramse abaixo ou próximo do valor de limite de quantificação do método. Também, os resultados encontrados estão em concentrações inferiores aos limites máximos permitidos preconizados em regulamentações internacionais $[32,33]$ e, deste modo, poderiam ser consideradas apropriadas para o consumo humano em âmbito nacional. 
Embora o objeto de estudo contemplasse somente os produtos dos tipos sem gás e sem adição de sais, e que a legislação nacional [5] atribua VMPs para as substâncias químicas $\mathrm{Ca}, \mathrm{K}, \mathrm{Mg}$ e $\mathrm{Na}$ somente para as águas quando adicionadas de sais, esses analitos também foram avaliados, e verificou-se que estes elementos apresentaram as maiores concentrações analisadas. $E$, ainda que o item 5.3.4 da legislação assinale que "A água adicionada de sais deverá conter no mínimo 30 $\mathrm{mg} / \mathrm{L}$ dos sais adicionados permitidos", quatro amostras analisadas ultrapassaram esse valor em pelo menos um dos seguintes elementos: Na para as amostras A, C e D e Ca para a amostra D. Este fato demonstra a ocorrência de diferenças de composição nas características geológicas do solo (hidrogeológica e hidrogeoquímica) existentes entre as distintas fontes de captação dessas águas, ou ainda às possíveis contribuições do clima, biota, entre outros fatores. Estes aspectos podem resultar em águas relativamente mais ricas em minerais e dependendo de sua procedência e nível de concentração, não devem afetar a saúde humana quando de seu consumo [25, 34].

As comparações dos resultados obtidos no presente estudo na avaliação das concentrações dos nutrientes e dos contaminantes inorgânicos em águas envasadas sem gás e sem adição de sais consumidas na cidade de São Paulo estão em concordância àqueles descritos em literatura nacional e internacional, conforme ilustrado na Tabela 2 [3, 26, 34-39]. É possível observar que as procedências das fontes de água nacionais para a captação de águas minerais envasadas avaliadas, apresentam baixa mineralização devido às características hidrogeológica e hidrogeoquímica do solo [25], semelhante à composição geológica da Romênia (país localizado no sudeste do continente europeu) [34].

Todos os fatos mencionados acima refletem a boa qualidade da água disponível no comércio da cidade de São Paulo, indicando a ausência de contaminação ambiental. Ainda, considerando o aumento no consumo da água mineral envasada no país, a diversidade de marcas presentes em outras localidades e à escassez de resultados recentes na literatura nacional que abordem sua composição química que possam atribuir valores de interações naturais ou ações antrópicas, este estudo conduz à continuidade de pesquisas e monitoramentos que atendam a promoção da saúde da população, em atendimento da Saúde Pública. 
Tabela 1: Valores máximos permitidos estabelecidos pela Resolução-RDC 274/2005 e os resultados das determinações das amostras de águas minerais comercializadas na cidade de São Paulo (A a J), por ICP-MS, para 18 elementos, em $\mathrm{mg} / \mathrm{L}$.

\begin{tabular}{|c|c|c|c|c|c|c|c|c|c|c|c|c|}
\hline Analito & $\begin{array}{c}* * \text { RDC } \\
274\end{array}$ & LQ & $\mathbf{A}$ & B & C & D & $\mathbf{E}$ & $\mathbf{F}$ & G & $\mathbf{H}$ & I & $\mathbf{J}$ \\
\hline Al & - & 0,0004 & $<\mathrm{LQ}$ & 0,004 & 0,007 & $<\mathrm{LQ}$ & $<\mathrm{LQ}$ & $<\mathrm{LQ}$ & $<\mathrm{LQ}$ & $<\mathrm{LQ}$ & $<\mathrm{LQ}$ & $<\mathrm{LQ}$ \\
\hline Ag & - & 0,002 & $<\mathrm{LQ}$ & $<\mathrm{LQ}$ & $<\mathrm{LQ}$ & $<\mathrm{LQ}$ & $<\mathrm{LQ}$ & $<\mathrm{LQ}$ & $<\mathrm{LQ}$ & $<\mathrm{LQ}$ & $<\mathrm{LQ}$ & $<\mathrm{LQ}$ \\
\hline As & 0,01 & 0,001 & 0,004 & $<\mathrm{LQ}$ & 0,007 & $<\mathrm{LQ}$ & $<\mathrm{LQ}$ & $<\mathrm{LQ}$ & $<\mathrm{LQ}$ & $<\mathrm{LQ}$ & $<\mathrm{LQ}$ & $<\mathrm{LQ}$ \\
\hline $\mathbf{B a}$ & 0,7 & 0,005 & 0,04 & 0,04 & 0,05 & 0,4 & 0,04 & 0,06 & 0,04 & 0,1 & 0,05 & 0,1 \\
\hline Be & - & 0,0004 & $<\mathrm{LQ}$ & $<\mathrm{LQ}$ & $<\mathrm{LQ}$ & $<\mathrm{LQ}$ & $<\mathrm{LQ}$ & $<\mathrm{LQ}$ & $<\mathrm{LQ}$ & $<\mathrm{LQ}$ & $<\mathrm{LQ}$ & $<\mathrm{LQ}$ \\
\hline $\mathbf{C a}$ & $* 250$ & 0,120 & 4,8 & 1,9 & 0,5 & 36,8 & 18,0 & 10,1 & 1,8 & 2,9 & $<\mathrm{LQ}$ & 19,0 \\
\hline Cd & 0,003 & 0,0005 & $<\mathrm{LQ}$ & $<\mathrm{LQ}$ & $<\mathrm{LQ}$ & $<\mathrm{LQ}$ & $<\mathrm{LQ}$ & $<\mathrm{LQ}$ & $<\mathrm{LQ}$ & $<\mathrm{LQ}$ & $<\mathrm{LQ}$ & $<\mathrm{LQ}$ \\
\hline $\mathrm{Cr}$ & 0,05 & 0,001 & $<\mathrm{LQ}$ & $<\mathrm{LQ}$ & 0,007 & 0,006 & 0,03 & 0,002 & 0,01 & 0,002 & 0,002 & 0,03 \\
\hline $\mathbf{C u}$ & 1 & 0,002 & 0,03 & 0,03 & 0,01 & 0,03 & 0,03 & $<\mathrm{LQ}$ & $<\mathrm{LQ}$ & $<\mathrm{LQ}$ & $<\mathrm{LQ}$ & $<\mathrm{LQ}$ \\
\hline $\mathbf{K}$ & $* 500$ & 0,030 & 1,1 & 1,2 & $<\mathrm{LQ}$ & 39,7 & 0,7 & 1,8 & 0,8 & 1,4 & 2,2 & 0,8 \\
\hline Mg & $* 65$ & 0,020 & 0,4 & 0,5 & 0,03 & 16,3 & 10,0 & 1,5 & 3,9 & 1,8 & 3,4 & 9,8 \\
\hline Mn & 0,5 & 0,025 & 0,03 & 0,03 & 0,03 & 0,03 & 0,03 & 0,03 & 0,03 & $<\mathrm{LQ}$ & $<\mathrm{LQ}$ & $<\mathrm{LQ}$ \\
\hline $\mathbf{N a}$ & $* 600$ & 0,023 & 34,2 & 1,4 & 71,7 & 39,5 & 0,7 & 2,6 & 1,9 & 2,8 & 2,0 & 0,8 \\
\hline Ni & 0,02 & 0,001 & $<\mathrm{LQ}$ & $<\mathrm{LQ}$ & $<\mathrm{LQ}$ & 0,001 & $<\mathrm{LQ}$ & $<\mathrm{LQ}$ & $<\mathrm{LQ}$ & $<\mathrm{LQ}$ & $<\mathrm{LQ}$ & $<\mathrm{LQ}$ \\
\hline $\mathbf{P b}$ & 0,01 & 0,001 & $<\mathrm{LQ}$ & $<\mathrm{LQ}$ & $<\mathrm{LQ}$ & $<\mathrm{LQ}$ & $<\mathrm{LQ}$ & $<\mathrm{LQ}$ & $<\mathrm{LQ}$ & $<\mathrm{LQ}$ & $<\mathrm{LQ}$ & $<\mathrm{LQ}$ \\
\hline Se & 0,01 & 0,001 & $<\mathrm{LQ}$ & $<\mathrm{LQ}$ & $<\mathrm{LQ}$ & $<\mathrm{LQ}$ & $<\mathrm{LQ}$ & $<\mathrm{LQ}$ & $<\mathrm{LQ}$ & $<\mathrm{LQ}$ & $<\mathrm{LQ}$ & $<\mathrm{LQ}$ \\
\hline Tl & - & 0,001 & $<\mathrm{LQ}$ & $<\mathrm{LQ}$ & $<\mathrm{LQ}$ & $<\mathrm{LQ}$ & $<\mathrm{LQ}$ & $<\mathrm{LQ}$ & $<\mathrm{LQ}$ & $<\mathrm{LQ}$ & $<\mathrm{LQ}$ & $<\mathrm{LQ}$ \\
\hline $\mathbf{Z n}$ & - & 0,001 & $<\mathrm{LQ}$ & $<\mathrm{LQ}$ & $<\mathrm{LQ}$ & $<\mathrm{LQ}$ & $<\mathrm{LQ}$ & $<\mathrm{LQ}$ & $<\mathrm{LQ}$ & 0,002 & 0,002 & $<\mathrm{LQ}$ \\
\hline
\end{tabular}

*Valores indicados para águas adicionadas de sais

***Resolução-RDC n'- 274 de 22 de setembro de 2005

LQ = limite de quantificação 
Tabela 2: Comparação entre os resultados obtidos no presente estudo e literatura, em mg/L.

\begin{tabular}{|c|c|c|c|c|c|c|c|c|c|}
\hline \multirow{2}{*}{ Analito } & \multirow{2}{*}{$\begin{array}{c}\text { Resultados } \\
\text { experimentais }\end{array}$} & \multicolumn{8}{|c|}{ Referência } \\
\hline & & 3 & 26 & 34 & 35 & 36 & 37 & 38 & 39 \\
\hline Al & $<0,004-0,007$ & $0,01-0,07$ & $0,08-4,08 *$ & - & - & - & - & - & - \\
\hline Ag & $<0,002$ & - & - & - & - & - & - & - & - \\
\hline As & $<0,001-0,007$ & - & $0,07-2,29 *$ & - & - & - & - & - & - \\
\hline $\mathbf{B a}$ & $0,04-0,1$ & $0,01-0,22$ & $0,04-90,55^{*}$ & - & - & - & - & $0,01-0,13$ & - \\
\hline $\mathrm{Be}$ & $<0,0004$ & - & - & - & - & - & - & - & - \\
\hline $\mathbf{C a}$ & $<0,210-36,8$ & $0,53-28,35$ & $0,6-41,7$ & 146 & - & $257,85-506,15$ & $0,13-150$ & $0,26-32,4$ & $1,221-31,778$ \\
\hline Cd & $<0,0005$ & - & - & - & $0,009-0,2$ & - & - & - & - \\
\hline $\mathrm{Cr}$ & $<0,001-0,03$ & $<0,005$ & $0,08-30,61 *$ & - & $0,002-0,62$ & - & - & - & - \\
\hline $\mathbf{C u}$ & $<0,002-0,03$ & - & $0,06-1,94 *$ & - & - & - & - & - & - \\
\hline $\mathbf{K}$ & $<0,030-39,7$ & $0,43-3,27$ & $0-9,1$ & 9,4 & - & $0,30-1,40$ & $0,27-7,4$ & $0,4-4,3$ & $0,148-3,632$ \\
\hline Mg & $0,03-16,3$ & $0,01-9,10$ & $1,0-29,8$ & 37,3 & - & $1,44-18,48$ & $0,03-100$ & $0,2-16,3$ & $0,223-8,478$ \\
\hline Mn & $<0,025-0,03$ & $0,04-0,04$ & $<0,06-1,46^{*}$ & - & - & - & - & $0,01-0,090$ & - \\
\hline $\mathbf{N a}$ & $0,7-39,5$ & $0,71-97,29$ & $0,1-85,2$ & 88 & - & $0,30-4,50$ & $2,64-200$ & $1,2-102,8$ & $0,276-6,652$ \\
\hline $\mathbf{N i}$ & $<0,001-0,001$ & - & $0,41-1,89 *$ & - & - & - & - & - & - \\
\hline $\mathbf{P b}$ & $<0,001$ & $<0,005$ & $<0,003-0,09^{*}$ & - & $<0,01$ & - & - & - & - \\
\hline Se & $<0,001$ & - & - & - & - & - & - & - & - \\
\hline Tl & $<0,001$ & - & - & - & - & - & - & - & - \\
\hline $\mathbf{Z n}$ & $<0,001-0,002$ & $<0,005-0,03$ & $<0,02-46,18^{*}$ & - & - & - & - & - & - \\
\hline
\end{tabular}

A tabela indica os valores de concentração dos elementos em estudo para as referências 3, 26, 34 a 39, transcritos conforme apresentados pelos autores

* Valores reportados pelos autores na unidade $\mu \mathrm{g} / \mathrm{L}$ 


\section{CONCLUSÃO}

Os resultados obtidos neste estudo na avaliação dos nutrientes e dos contaminantes inorgânicos em água mineral envasada, consumida na cidade de São Paulo, propicia o fornecimento de subsídios para futuras pesquisas no estabelecimento de valores máximos permitidos para os parâmetros ainda não previstos em legislação $(\mathrm{Ag}, \mathrm{Al}, \mathrm{Be}, \mathrm{Tl}$ e $\mathrm{Zn})$, uma vez que a presença desses metais e semimetais em níveis de concentrações elevados pode ser tóxica ao organismo e causar efeitos prejudiciais à saúde humana; de forma semelhante aos nutrientes previstos somente para as águas adicionadas de sais $(\mathrm{Ca}, \mathrm{K}, \mathrm{Mg}$ e $\mathrm{Na})$.

Portanto, considerando o amplo consumo popular de água mineral envasada e seu potencial risco à saúde, bem como a importância da água em termos ambientais em seus diversos usos e o impacto que os metais e semimetais podem acarretar sobre a Saúde Pública quando presentes nessa matriz, é possível inferir sobre o mérito da implementação do monitoramento continuado do Programa de Alimentos em demais localidades do Estado de São Paulo. Os resultados analíticos emitidos pelo laboratório proporcionam ferramenta às autoridades sanitárias locais na identificação de problemas relacionados à produção e/ou comercialização e na tomada de ação de intervenção de caráter preventivo, visando avaliar os impactos sobre os riscos à saúde da população.

\section{AGRADECIMENTOS}

Agradecimentos à equipe técnica do Núcleo de Contaminantes Inorgânicos do Instituto Adolfo Lutz, que contribuíram para o desenvolvimento deste trabalho.

\section{REFERÊNCIAS BIBLIOGRÁFICAS}

1. Tohanean DI, Koronas V, Sagat P, Turcu I, Curitianu IM, Alexe CI, et al. Study on water and other drinks used for hydration in movement activities. Rev Chim. 2020;71(8):249-54. doi: 10.37358/Rev.Chim.1949

2. Stoots SJM, Kamphus GM, Geraghty R, Vogt L, Henderickx MMEL, Hameed BMZ, et al. Global variations in the mineral content of bottled still and sparkling water and a description of the possible impact on nephrological and urological diseases. J Clin Med. 2021;10:2807. doi: 10.3390/jcm10132807

3. Morgano MA, Schatti AC, Enriques HA, Mantovani DMB. Avaliação físico-química de águas minerais comercializadas na região de Campinas, SP. Ciênc Tecnol Aliment. 2002 Set-Dez;22(3):239-43. doi: 10.1590/S0101-20612002000300007

4. Mocek-Płóciniak A, Skowrońska M. Water - an important element not only of the soil environment. Soil Sci Ann. 2021;72(1):134620. doi: 10.37501/soilsa/134620

5. Brasil. Ministério da Saúde, Agência Nacional de Vigilância Sanitária. Resolução - RDC n 274 , de 22 de setembro de 2005. Aprova o Regulamento técnico para águas envasadas e gelo. Diário Oficial da União. 23 set 2005;184(Seção 1):376-377. Disponível em: https://bvsms.saude.gov.br/bvs/saudelegis/anvisa/2005/res0274-22_09_2005.html

6. Ribeiro TI, Rocha Filho LB, Barros FAA, Barros SBA. Avaliação físico-química de águas minerais comercializadas em Picos, Piauí, Brasil. Braz J Dev. 2021;7(2):15566-79. doi: 10.34117/bjdv7n2-262

7. Machado RHL, Marinho LFC, Dworak ES, Carolino IDR, Conegero CI, Massago M. Água mineral comercializada no Brasil: Caracterização, consumo e qualidade microbiológica. Arq Mudi. 2021;25(1):12-25. doi: 10.4025/arqmudi.v25i1.56828

8. Oliveira BTA, Oliveira CV, Felippe MF. Influência dos depósitos de rejeito de mineração nas características químicas das águas: estudos na planície tecnogênica do Alto do Rio Doce, MG. Cadernos de Geografia. 2021;31(1):70-89. doi: 10.5752/p.2318-2962.2021v31nesp1p70

9. Health Canada. Guidelines for canadian drinking water quality. Summary table. Water and air quality Bureau, healthy environments and consumer safety branch, health Canada. Ottawa (CA): Government of Canada [Internet]; 2020 [citado em 24 jun 2021]. Disponível em: https://www.canada.ca/en/healthcanada/services/environmental-workplace-health/reports-publications/water-quality.html 
10. World Health Organization (WHO). Guidelines drinking-water quality: Fourth edition incorporation the first addendum [Internet]. Geneva (CH): WHO; 2017 [citado em 24 jun 2021]. Disponível em: https://apps.who.int/iris/bitstream/handle/10665/254637/9789241549950-eng.pdf

11. Balali-Mood M, Naseri K, Tahergorabi Z, Khazdair MR, Sadeghi M. Toxic mechanisms of five heavy metals: Mercury, lead, chromium, cadmium, and arsenic. Front Pharmacol. 2021;12:643972. doi: 10.3389/fphar.2021.643972

12. Secretaria de Estado da Saúde de São Paulo, Centro de Vigilância Sanitária [Internet]. Programas e Projetos. Programa paulista de alimentos; [data não referenciada; citado em 25 jun 2021]. Disponível em: http://www.cvs.saude.sp.gov.br/prog_det.asp?te_codigo $=1 \&$ pr_codigo $=6$

13. Skoog DA, West DM, Holler FJ, Crouch SR. Fundamentos de química analítica. Tradução da $9^{a}$ edição. São Paulo (SP): Editora Cengage Learning Brasil; 2014.

14. Secretaria de Estado da Saúde de São Paulo, Centro de Vigilância Sanitária. Programa paulista de análise fiscal de alimentos 2018 ( $2^{\circ}$ Semestre) [Internet]; 2018 [citado em 25 jun 2021]. Disponível em: http://www.cvs.saude.sp.gov.br/up/Instru\%C3\%A7\%C3\%B5es\%20Programa\%20Paulista\%20de\%20 Alimentos\%202\%C2\%BA\%20sem\%202018.pdf

15. Secretaria de Estado da Saúde de São Paulo, Centro de Vigilância Sanitária. Instruções Programa paulista de análise fiscal de alimentos 2019 ( $1^{\circ}$ Semestre) [Internet]; 2019 [citado em 25 jun 2021]. Disponível em: http://www.cvs.saude.sp.gov.br/up/Instru\%C3\%A7\%C3\%B5es\%20PROGRAMA\%20 PAULISTA\% $20 \% 202 \% \mathrm{C} 2 \% \mathrm{BA} \% 20 \mathrm{sem} \% 202019$.pdf

16. Association of Official Agricultural Chemists (AOAC). Official Methods of Analysis of AOAC International [Internet]. Rockville (MD): AOAC; 2016. Appendix F, Guidelines for standard method performance requeriments; [citado em 24 jun 2021]; p. 1-18. Disponível em: http://www.eoma.aoac.org/app_f.pdf

17. Arauz LJ, Buzzo ML, Mataveli LRV, Tiglea P. Avaliação da efetividade de participações em programas de ensaio de proficiência no controle da qualidade dos resultados analíticos na determinação de metais e semimetais em águas em laboratório de Saúde Pública. Infarma. 2019;31(3):177-86. doi: 10.14450/2318-9312.v31.e3.a2019.pp177-186

18. Creed JT, Brockhoff CA, Martin TD. Method 200.8: Determination of trace elements in waters and waste by inductively coupled plasma-mass spectrometry [Internet]. Revision 5.4. Cincinnati (OH): U.S. Environmental Protection Agency; 1994 [citado em 26 mar 2021]. Disponível em: https://yosemite.epa.gov/oa/eab_web_docket.nsf/Attachments\%20By\%20ntFilingId/482881CDAF52 A73985257D55005D9BEB/\$FILE/EPA\%20Method\%20200.8\%20(00608866xB76D6).pdf

19. Associação Brasileira de Normas Técnicas (ABNT). ABNT NBR ISO/IEC 17025:2017: Requisitos gerais para competência de laboratórios de ensaio e calibração. Rio de Janeiro: ABNT; 2017.

20. Instituto Nacional de Metrologia, Qualidade e Tecnologia (INMETRO). Escopo da acreditação ABNT NBR ISO/IEC 17025 - Ensaio. Acreditação n ${ }^{\circ}$ CRL 0679 [Internet]. Brasília (DF): INMETRO; c1993-2012 [citado em 26 mar 2021]. Disponível em: http://www.inmetro.gov.br/laboratorios/rble/docs/CRL0679.pdf

21. Associação Brasileira das Indústrias de Refrigerantes e de Bebidas Não Alcoólicas (ABIR) [Internet]. Águas Minerais. Volume de produção do mercado brasileiro de águas minerais dos anos de 2010 a 2019; c2015 [citado em 25 jun 2021]. Disponível em: https://abir.org.br/o-setor/dados/aguas-minerais/

22. Pulzatto ME, Specian AM, Souza GR. Avaliação sensorial de marcas comerciais de água mineral sem gás. FTT Journal Engeneering and Business. 2016;(1):42-58. Disponível em: http://journal.ftt.com.br/seer/index.php/FTT/article/view/14

23. Pacheco MHS, Kuriya SP, XCapobiango CSC, Pimentel TC, Cruz AG, Esmerino EA, et al. Exploration of gender diferences in bolttled mineral water consumption: A projective study of consumer's perception in Brazil. J Sens Stud. 2018;33:e12434. doi:101111/joss.12434

24.Zapata $\mathrm{O}$. The relationship between climate conditions and consumption of bottled water: A potential link between climate change and plastic pollution. Ecol Econ. 2021;187:107090. doi: 10.1016/j.ecolecon.2021.107090

25. Bulia IL, Enzweiler J. The hydrogeochemistry of bottled mineral water in São Paulo state, Brazil. J Geochem Explor. 2018;188:43-54. doi: 10.1016/j.gexplo.2018.01.007

26. Deng L, Xu B, Yang X, Hu A. Water quality and health risk assessment based on hydrochemical characteristics of tap and large-size bottled water from the main cities and towns in Guanzhong Basin, China. Environ Earth Sci. 2021;80:139. doi: 10.1007/s12665-021-09415-x

27. Jesus LS, Riyis MT, Giacheti HL. Fontes potenciais de poluição das águas subterrâneas e uma proposta de priorização de áreas de fiscalização em um município industrializado. Águas Subterrâneas. 2021;35(2):136-47. doi: 10.14295/ras.v35i2.30028 
28. Silva NBD, Borges I, Vasconcelos VV. Análise do impacto potencial de áreas com contaminação de solos sobre cursos d'água e poços no município de São Paulo. Águas Subterrâneas. 2020;34(3):250-63. doi: $10.14295 /$ ras.v34i3.29753

29. Oliveira ED, Martins TCD, Santos WR, Serra MC, Pionheiro EM. Análise mercadológica de água mineral engarrafada em São Luís - MA. Qualitas Rev Eletr. 2018;19(3):38-52. doi: 10.18391/req.v19i3.3208

30. Reis LR, Bevilacqua PD, Carmo RF. Água envasada: Qualidade microbiológica e percepção dos consumidores no município de Viçosa (MG). Cad Saúde Colet. 2014;22(3):224-32. doi: 10.1590/1414462X201400030002

31. Aslani H, Pashmtab P, Shaghaghi A, Mohammadpoorasl A, Taghipour H, Zarei M. Tendencies towards bottled drinking water consumption: Challenges ahead of polyethylene terephthalate (PET) waste management. Health Promot Perspect. 2021 Feb;11(1):60-8. doi: 10.34172/hpp.2021.09

32. U.S. Food \& Drug Administration (FDA) [Internet]. CFR - Code of Federal Regulations. Title 21 Food and drugs. Vol. 2, Part 165, Sec. 165.110; 01 abr 2020 [cited 2021 mar 10]. Available from: https://www.accessdata.fda.gov/scripts/cdrh/cfdocs/cfcfr/CFRSearch.cfm?fr=165.110

33. Ministerio de Salud, ANMAT. Código Alimentario Argentino [Internet]. Argentina: ANMAT; 2012. Capítulo XII: Bebidas hídricas, agua y agua gasificada, Art. 985 - (Resolución Conjunta SCS y SAByDR N ${ }^{\circ}$ 2/2021); [atualizado em jan 2021; citado em 16 mar 2021]. [p. 7-9]. Disponível em: https://www.argentina.gob.ar/sites/default/files/caa_capitulo_xii_aguas_actualiz_2021-01.pdf

34. Bodor K, Bodor Z, Szép A, Szép R. Classification and hierarchical analysis of principal Romanian bottled mineral Waters. J Food Compost Anal. 2021 Jul;100:103903. doi: 10.1016/j.jfca.2021.103903

35. Banda RK, Mubita P, Moonga G, Meki CD. Bacteriological quality and heavy metal analysis of packaged water produced in Lusaka, Zambia and associated quality control measures. Front Public Health. 2021;9:620700. doi: 10.3389/fpubh.2021.620700

36. Najah ZM, Salem BA, Aburas NM. Analysis of some bottled drinking water samples available in Alkoms city. J Academ Res. 2021;17:22-5.

37. Iyakare JD, Taupin JD, Hitimana CN, Dusabimana T, Ghalit M, El Ouahabi M, et al. Hydrochemical study of bottled water in Rwanda and relationship with their origin. Water Supply. 2021 Jul;ws2021211. doi: $10.2166 /$ ws.2021.211

38. de Maio FD, Okada IA, Carvalho MFH, Kira CS, Duran MC, Zenebon O. Avaliação da rotulagem e dos teores de minerais e contaminantes inorgânicos em águas minerais nacionais e importadas. Rev Inst Adolfo Lutz. 2002;61(1):27-32.

39. Maro KF, Pires CV, Junqueira MS, Macedo MCC, Silva LS. Análises físico-químicas e microbiológicas de amostras de água mineral. Res, Soc Dev. 2020;9(8):e940986581. doi: 10.33448/rsd-v9i8.6581 